\title{
A Modified Four Path Method for Calculating Coupling Field of Super-Low Altitude Aircraft Target
}

\author{
Gaoxiang Zou' ${ }^{1}$, Chuangming Tong1, Binglin $\mathrm{Yu}^{2}$, Hualong Sun${ }^{1}$, Tao Song1 \\ ${ }^{1}$ Air Force Engineering University, Xi'an, China \\ ${ }^{2}$ The 95226 Army of the Chinese People's Liberation Army, Wuhan, China \\ Email: gxiangzou@163.com
}

How to cite this paper: Zou, G.X., Tong, C.M., Yu, B.L., Sun, H.L. and Song, T. (2018) A Modified Four Path Method for Calculating Coupling Field of Super-Low Altitude Aircraft Target. Journal of Computer and Communications, 6, 64-73. https://doi.org/10.4236/jcc.2018.612006

Received: September 14, 2018 Accepted: December 23, 2018 Published: December 26, 2018

\begin{abstract}
A novel modified four path method (FPM) is presented for calculating coupling field of super-low altitude aircraft target. Based on the hybrid method PO + MEC (Physical Optics and Method of Equivalent Currents), the antenna radiation pattern is introduced to consider the multipath interference from side lobe of seeker. The modified FPM is used to calculate the coupling field from super-low altitude aircraft target above different terrestrial environments. The curves of scattering coefficient are analyzed. The influences of height of target, root mean square (RMS), and incident angle on coupling field characteristics are discussed. The simulation results can be used for reference in detection for super-low altitude target and optimization for radar system.
\end{abstract}

\section{Keywords}

Modified Four Path Method, Antenna Radiation Pattern, Super-Low Altitude, Aircraft Target, Coupling Field, Brewster Angle

\section{Introduction}

The scattering characteristics of aircraft target above terrestrial environments are complex and variable. The scattering characteristics often changes with many factors such as direction of detection, position of target and parameters of environment. The composite scattering problems [1] [2] [3] [4] [5] of target above environment have become a research hotpot in the field of computational electromagnetic. The multipath interference in the detection for super-low altitude target is usually described by coupling field. The FPM [6] [7] proposed by J. T. 
Johnson is suitable for extracting coupling field. However, the multipath interference from side lobe of seeker cannot be neglected. Therefore, the effect of side lobe must be considered.

This paper attempts to establish a kind of scattering model for super-low altitude aircraft target. Based on the traditional FPM, the Gauss antenna radiation pattern is introduced to modify the FPM model. The hybrid method PO + MEC is applied to calculate the scattering from facets and edges of target. Some examples are given by using the modified FPM, and some curves of coupling field with some factors are analyzed. From the numerical results, the characteristics of coupling field can be concluded which can be used for reference in detection for super-low altitude target and optimization for radar system.

\section{Modified Four Path Method}

The detection for super-low altitude aircraft target above environment belongs to the composite scattering problem of computational electromagnetics. The FPM proposed by J. T. Johnson is used to analyze the scattering from simple target above plane. However, traditional FPM cannot count the multipath interference which enters the radar/seeker through side lobe. The scattering energy of multipath interference which the radar/seeker receives through main lobe can have a stronger effect than that through side lobe. Therefore, aimed at counting the effect of antenna radiation pattern, the Gauss antenna radiation pattern is introduced. We set one main lobe and two side lobes beside it respectively. And we set the gain of side lobe as $g_{3}$. The function of Gauss antenna radiation pattern is taken in the form:

$$
G_{M B}(\theta)=\exp \left(-2 \ln 2 \theta^{2} / \theta_{B}^{2}\right),|\theta| \leq \mu
$$

where $\theta_{B}$ denotes half-power beam point width; $\mu$ denotes azimuth angle when the main lobe's gain is set as $g_{3}$ which is defined as follow:

$$
\mu=\theta_{B} \sqrt{\ln g_{3} /(-2 \ln 2)}
$$

The antenna radiation pattern of first side lobe can be described by the Gauss function at central position of $\pm 1.5 \mu$, which is taken in the form:

$$
G_{B 1}(\theta)=g_{1} \exp \left(\frac{-2 \ln 2(\theta+1.5 \mu)^{2}}{\theta_{B 1}^{2}}\right), \mu \leq|\theta| \leq 2 \mu
$$

where $g_{1}$ is the peak gain of the first side lobe; $\theta_{B 1}$ is taken in the form:

$$
\theta_{B 1}=0.5 \mu \sqrt{(-2 \ln 2) / \ln \left(g_{3} / g_{1}\right)}
$$

The antenna radiation pattern of second side lobe can be described by the Gauss function at central position of $\pm 2.5 \mu$, which is taken in the form:

$$
G_{B 2}(\theta)=g_{2} \exp \left(\frac{-2 \ln 2(\theta \pm 2.5 \mu)^{2}}{\theta_{B 2}^{2}}\right), 2 \mu<|\theta| \leq 3 \mu
$$

where 


$$
\theta_{B 2}=0.5 \mu \sqrt{(-2 \ln 2) / \ln \left(g_{3} / g_{2}\right)}
$$

In this paper, he peak gain of the first side lobe is $G_{1}=10 \log _{10} g_{1}=-20 \mathrm{~dB}$; The peak gain of the second side lobe is $G_{2}=10 \log _{10} g_{2}=-25 \mathrm{~dB}$; The peak gain of other side lobe is $G_{r}=10 \log _{10} g_{3}=-30 \mathrm{~dB}$; The corresponding gain is $g_{1}=0.01, g_{2}=10^{-2.5}=0.00316$ and $g_{3}=0.001$ respectively.

In Figure 1, $H$ and $h$ denote the height of target and seeker respectively; $R$ denotes the spatial distance between target and seeker; $D$ denotes the distance between target's and seeker's projector point on the ground. The parameters in Figure 1 can be calculated by the equations as follow:

$$
\left\{\begin{array}{l}
h=R \cos \theta_{1}+H \\
D=R \sin \theta_{1} \\
\theta_{2}=\theta_{3}=\tan ^{-1}[D /(H+h)] \\
\theta_{4}=\theta_{1}
\end{array}\right.
$$

In Tradition FPM, the surface with small roughness is simplified as infinite smooth plane, and the reflection energy is approximate to concentrating in the direction of specular reflection. However, when surface becomes rougher, the diffuse reflection will become dominant. Hence, we use the modified complex reflection coefficient to describe the effect of environment which is taken in the form:

$$
\rho\left(\theta_{i}^{\text {sur }}\right)=\Gamma\left(\theta_{i}^{\text {sur }}\right) \rho_{\mathrm{s}}\left(\theta_{i}^{\text {sur }}\right)
$$

Here $\theta_{i}^{\text {sur }}$ is the incident angle illuminating the environmental surface; $\Gamma$ denotes the Fresnel reflection coefficient; $\rho_{\mathrm{s}}$ denotes the reflection factor of rough surface. We set the polarization mode as Vertical-to-Vertical (VV) in this paper.

Set $\boldsymbol{E}\left(\theta_{i}, \theta_{s}\right)$ and $\boldsymbol{E}^{\prime}\left(\theta_{i}, \theta_{s}\right)$ as scattering field from target and mirror target respectively. $\theta_{i}$ and $\theta_{s}$ denote incident angle and scattering angle respectively. According to Figure 1, the propagation path of electromagnetic wave in every path can be summarized as follow:

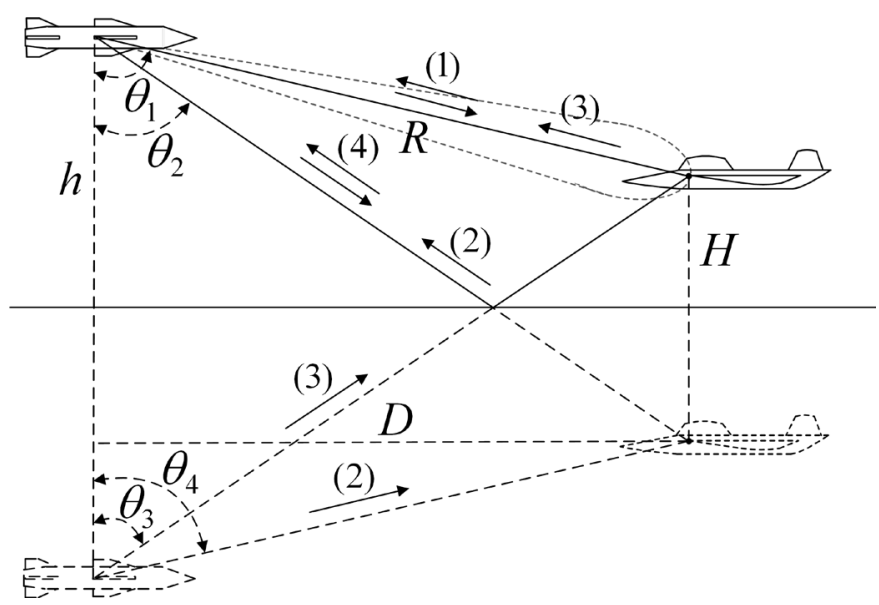

Figure 1. Model of modified four path method. 
1) The first path is "seeker $\rightarrow$ target $\rightarrow$ seeker". The scattering field of target is $\boldsymbol{E}_{1}\left(\theta_{1}, \theta_{1}\right)$.

2) The second path is "seeker $\rightarrow$ target $\rightarrow$ environment $\rightarrow$ seeker". The equivalent path is "mirror seeker $\rightarrow$ mirror target $\rightarrow$ environment $\rightarrow$ seeker". The scattering field of mirror target is $\rho\left(\theta_{2}\right) \boldsymbol{E}_{2}^{\prime}\left(\pi-\theta_{4}, \theta_{2}\right)$.

3) The third path is "seeker $\rightarrow$ environment $\rightarrow$ target $\rightarrow$ seeker". The equivalent path is "mirror seeker $\rightarrow$ environment $\rightarrow$ target $\rightarrow$ seeker". The scattering field of target is $\rho\left(\theta_{2}\right) \boldsymbol{E}_{3}\left(\pi-\theta_{3}, \theta_{1}\right)$.

4) The fourth path is "seeker $\rightarrow$ environment $\rightarrow$ target $\rightarrow$ environment $\rightarrow$ seeker". The equivalent path is "seeker $\rightarrow$ environment $\rightarrow$ mirror target $\rightarrow$ environment $\rightarrow$ seeker". The scattering field of mirror target is $\rho^{2}\left(\theta_{2}\right) \boldsymbol{E}_{4}^{\prime}\left(\theta_{2}, \theta_{2}\right)$.

According to the scattering mechanism in Figure 1, the multipath interference of Path 3 enters in seeker through the main lobe. However, the multipath interference of Path 2 and Path 4 enters in seeker through the side lobe. Hence, this discrimination must be considered. We set $\Delta \theta$ as included angle between the direction of main lobe's maximum gain and the direction of multipath interference from environment. Set the main lobe's maximum gain as $\left.G_{M B}(\theta)\right|_{\theta=0^{\circ}}=1$. The correction factor which is used to describe this kind of side lobe effect is given by the form as follow:

$$
\xi(\Delta \theta)=\frac{G(\Delta \theta)}{\left.G_{M B}(\theta)\right|_{\theta=0^{\circ}}}=\left\{\begin{array}{l}
G_{M B}(\Delta \theta),|\Delta \theta| \leq \mu \\
G_{B 1}(\Delta \theta), \mu \leq|\Delta \theta| \leq 2 \mu \\
G_{B 2}(\Delta \theta), 2 \mu \leq|\Delta \theta| \leq 3 \mu
\end{array}\right.
$$

Based on traditional FPM model in Figure 1, combined with correction factor in Equation (9), the modified FPM is given by the form as follow:

$$
\begin{aligned}
\boldsymbol{E}^{s}= & \boldsymbol{E}_{1}\left(\theta_{1}, \theta_{1}\right)+\xi(\Delta \theta) \rho\left(\theta_{2}\right) \boldsymbol{E}_{2}^{\prime}\left(\pi-\theta_{4}, \theta_{2}\right)+\rho\left(\theta_{2}\right) \boldsymbol{E}_{3}\left(\pi-\theta_{3}, \theta_{1}\right) \\
& +\xi(\Delta \theta) \rho^{2}\left(\theta_{2}\right) \boldsymbol{E}_{4}^{\prime}\left(\theta_{2}, \theta_{2}\right)
\end{aligned}
$$

Here $\boldsymbol{E}^{\boldsymbol{s}}$ denotes the electric field vector which the seeker receives in total. Except for Path 1, the sum of electric field vector for other three paths is called as multipath interference, which has great effect on detection for super-low altitude target.

\section{Formulation}

\subsection{Physical Optics (PO)}

We establish a simulation model for a kind of aircraft and subdivide it into many triangle facets by using Hypermesh software. The PO method [8] is used to calculate the far field from every facet of aircraft model. The PO integral is taken in the form:

$$
\boldsymbol{E}^{\mathrm{facet}} \approx \frac{i k_{0}}{4 \pi} \frac{e^{-j k_{0} r}}{r}\left[\hat{\boldsymbol{s}} \times\left(\boldsymbol{M}+\eta_{0} \hat{\boldsymbol{s}} \times \boldsymbol{J}\right)\right] \cdot \Delta \mathrm{A} \cdot I
$$

Here $\boldsymbol{J}$ and $\boldsymbol{M}$ denotes electric current and magnetic current of the facet respectively; $\Delta \mathrm{A}$ denotes the area of triangle facet. Based on the Gardon integral, 
$I$ is taken in the form:

$$
I=\frac{1}{j k_{0}|\hat{\boldsymbol{n}} \times \boldsymbol{w}| \Delta A} \sum_{m=1}^{3}(\hat{\boldsymbol{n}} \times \boldsymbol{w}) \cdot \boldsymbol{a}_{m} \exp \left(j k_{0} \boldsymbol{r}_{m} \cdot \boldsymbol{w}\right) \sin c\left(\frac{1}{2} k_{0} \boldsymbol{a}_{m} \cdot \boldsymbol{w}\right)
$$

In Equation (12), $\boldsymbol{w}=\hat{\boldsymbol{s}}-\hat{\boldsymbol{i}}, \sin c(x)=\sin x / x ; \boldsymbol{a}_{m}$ is a vector of the $\mathrm{m}$-th edge structure; $\boldsymbol{r}_{\boldsymbol{m}}$ is position vector for midpoint of the $\mathrm{m}$-th edge structure; $\hat{\boldsymbol{i}}$ and $\hat{\boldsymbol{s}}$ denotes unit vector of incident and scattering direction respectively; $k_{0}$ and $\eta_{0}$ are wave number and wave impedance in free space respectively; When $|\hat{\boldsymbol{n}} \times \boldsymbol{w}|=0$, Equation (12) can be written as follow:

$$
I=\exp \left(j k r_{0} \cdot \boldsymbol{w}\right)
$$

In Equation (13), $\boldsymbol{r}_{0}$ denotes position vector of arbitrary point belonging to the facet $i$. In this paper, we set that point as mass center of the facet $i$. Finally, the far field of all facets can be obtained by adding every facet's far field.

\subsection{Method of Equivalent Currents (MEC)}

The aircraft model contains several edge structures. The scattering from these edge structures cannot be neglected. We introduce the theory of equivalent electric current [9] which was proposed by Michaeli to calculate the edge diffraction. Set the edge as $C$, the far field of edge diffraction is obtained by substitute equivalent electric current into the radiation integral, which is taken in the form:

$$
\boldsymbol{E}^{\text {edge }}=\frac{i k_{0}}{4 \pi} \frac{e^{-j k_{0} r}}{r} \int_{C}\left[\eta_{0} \hat{\boldsymbol{s}} \times(\hat{\boldsymbol{s}} \times \boldsymbol{J})+\hat{\boldsymbol{s}} \times \boldsymbol{M}\right] e^{i k_{0} \hat{s} \cdot r^{\prime}} d l
$$

In which $\boldsymbol{J}$ and $\boldsymbol{M}$ present equivalent electric current and equivalent magnetic current of edge $C$ respectively; $\hat{s}$ denotes unit vector of observation direction; $\boldsymbol{r}^{\prime}$ denotes a vector from original point to certain point located in edge $C$; $\mathrm{d} l$ denotes arc length increment of edge $C$.

\section{Numerical Results}

In this section, the characteristics of coupling field from super-low altitude aircraft target above different terrestrial environments are analyzed by modified FPM. The influences of height of target, root mean square (RMS), and incident angle on coupling field characteristics are discussed.

Firstly, we explain the simulation conditions of following computation examples: working frequency is set as $10 \mathrm{GHz}$; Polarization mode of illuminating wave is VV polarization; Relative dielectric constant of concrete surface equals to 3.0-j0.00036; Relative dielectric constant of dry land surface equals to $4.0-\mathrm{j} 0.00018$; Relative dielectric constant of wet land surface equals to $30.0-\mathrm{j} 0.36$; The root mean height (RMS) of rough surface is $0.1 \lambda$ in all computation examples except for example 4.3; The target model is set as perfect conductor aircraft; The nose of aircraft is facing the positive direction of $\mathrm{x}$ axis; The height of aircraft is 15 min all computation examples except for example 4.4; The spatial distance between seeker and target is set as $5 \mathrm{~km}$; The half-power beam width of seeker is 
$5^{\circ}$; The observation angle ranges from $0^{\circ}$ to $90^{\circ}$ (corresponding grazing angle $\phi_{s}$ ranges from $90^{\circ}$ to $0^{\circ}$ ).

\subsection{Comparison of Different Terrestrial Environments}

Based on the modified FPM, the curves of coupling field from super-low altitude aircraft target above different terrestrial environments are obtained as follow.

In Figure 2, the curve of coupling field reaches the minimum value at the angle of $30^{\circ}$, and this angle is the Brewster angle of concrete surface. Equally, the curves of Figure 3 and Figure 4 reach the minimum value at the angle of $26.5^{\circ}$ and $10^{\circ}$ respectively. They are also the Brewster angles of corresponding surfaces. According to the numerical results, the coupling effect between target and environment is weakest at the angle of Brewster angle. If the seeker detects the super-low altitude aircraft target at the Brewster angle, the multipath interference has a minimal impact on the detection.

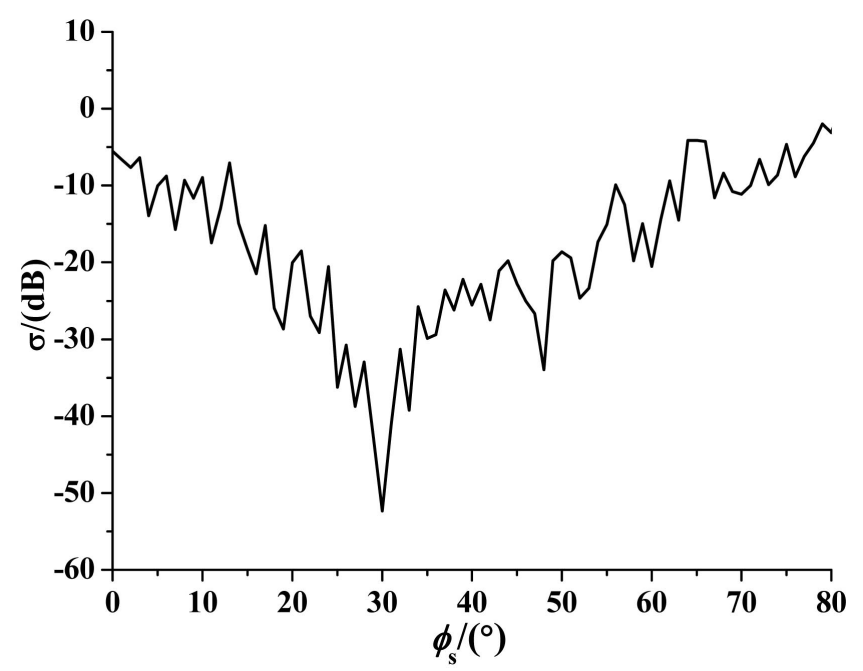

Figure 2. Coupling field of super-low altitude aircraft target above concrete surface.

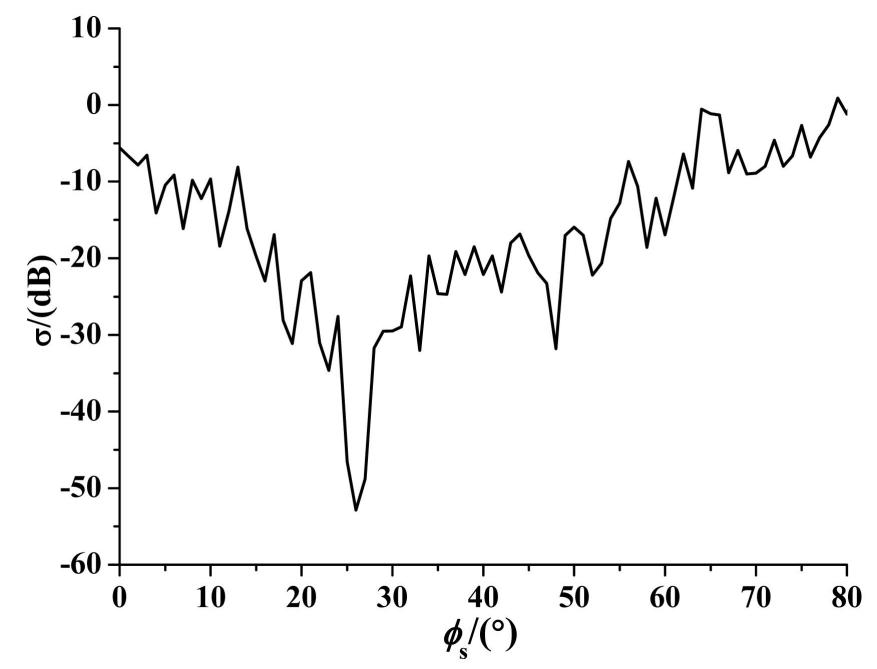

Figure 3. Coupling field of super-low altitude aircraft target above dry land surface. 


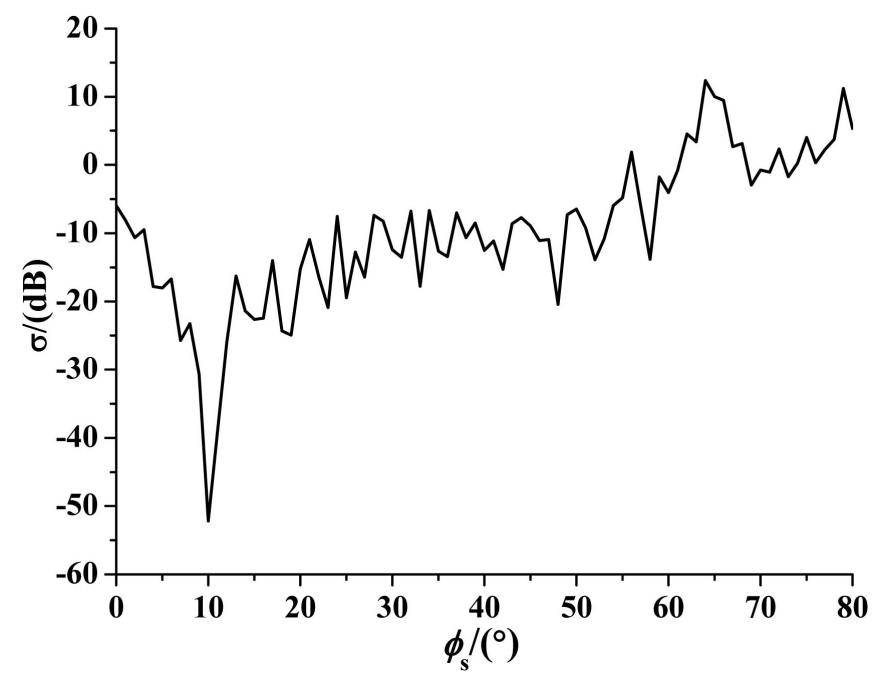

Figure 4. Coupling field of super-low altitude aircraft target above wet land surface.

\subsection{Azimuth Distribution Characteristic of Coupling Field}

In this computation example, the azimuth distributions of coupling field with different incident angle are calculated. Set terrestrial surface as concrete surface. Set the incident angles $\theta_{i}$ as $45^{\circ}, 60^{\circ}$ and $75^{\circ}$ (corresponding grazing angles $\phi_{\text {in }}$ are $45^{\circ}, 30^{\circ}$ and $15^{\circ}$ ). The curves of azimuth distribution with different incident angle are given as shown in Figure 5. In Figure 5, there is an obvious peak value existing at the azimuth angle of $180^{\circ}$ in every curve. The phenomenon shows that the multipath interference is strongest if the seeker detects the target along the direction of the tail. As shown in Figure 5, when the seeker detects the target at the grazing angle $30^{\circ}$, the coupling field is weaker than the curve with other incident angle. The reason causing this phenomenon is the Brewster effect. The Brewster angle of concrete surface is $30^{\circ}$, therefore, when electromagnetic wave illuminates the surface at the Brewster angle in VV polarization, the specular scattering will reach the minimum level. By contrast, when seeker detects the target at the grazing angle $45^{\circ}$ or $15^{\circ}$, the azimuth distributions of coupling field are at the similar level. From the numerical results, a conclusion is obtained that if the seeker detects target at the Brewster angle, the multipath interference will be weakened in azimuth distribution.

\subsection{Coupling Field with Different RMS}

In this computation example, the influences of RMS on coupling field are discussed. Set terrestrial surface as concrete surface. Set the RMS $h_{r m s}$ as $0.1 \lambda, 0.3 \lambda$ and $0.5 \lambda$. The curves of coupling field with different RMS are as shown as follow.

In Figure 6, with the increase of RMS, the coupling field of almost angles decreases obviously. The reason causing the phenomenon is that when the environmental surface becomes rougher, the diffuse scattering characteristic of the environment enhances that leads to the weaker multipath interference. 


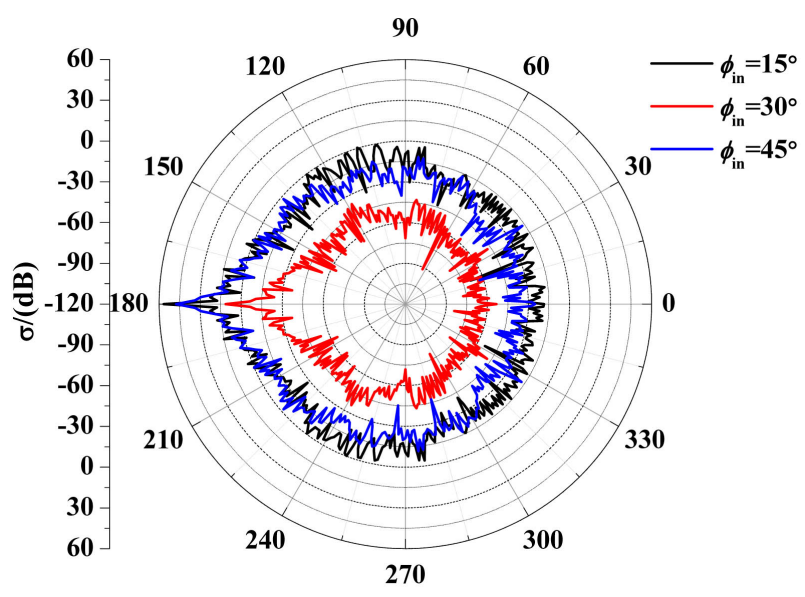

Figure 5. Azimuth distribution of coupling field with different incident angle.

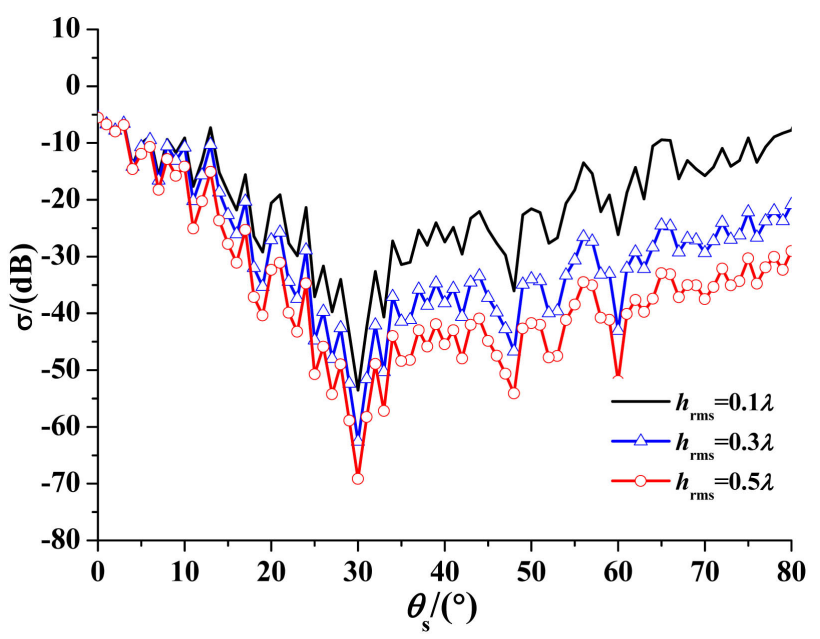

Figure 6. Coupling field with different RMS.

\subsection{Coupling Field with Different Target Height}

In this computation example, the influences of target height on coupling field are discussed. Set terrestrial surface as concrete surface. Set the target height as $15 \mathrm{~m}, 30 \mathrm{~m}, 45 \mathrm{~m}, 60 \mathrm{~m}, 75 \mathrm{~m}$ and $90 \mathrm{~m}$. The curves of coupling field with different target height are given as follow.

In Figure 7, with the increase of target height, the coupling field of almost angles decreases gradually. The reason causing the phenomenon is that when the target height becomes higher, the coupling effect between target and environment becomes weaker. When target height is $15 \mathrm{~m}$ or $30 \mathrm{~m}$, the corresponding angle of minimum value is $30^{\circ}$. When target height is $45 \mathrm{~m}$, the corresponding angle of minimum value is $29.5^{\circ}$. When target height is $60 \mathrm{~m}$, the corresponding angle of minimum value is $29^{\circ}$. When target height is $75 \mathrm{~m}$ or $90 \mathrm{~m}$, the corresponding angle of minimum value is $28^{\circ}$. A conclusion is obtained that with the increase of target height, the corresponding angle of minimum value for coupling field decreases. Therefore, when seeker detects target at different height, the detection angle must change correspondingly. 


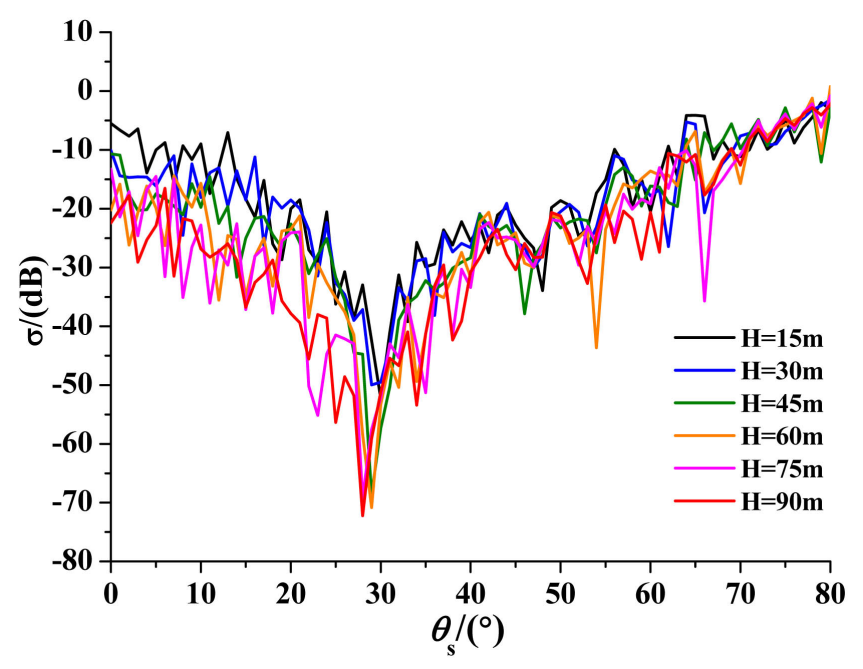

Figure 7. Coupling field with different target height.

\section{Conclusion}

This paper attempts to establish a kind of scattering model for super-low altitude aircraft target. Based on the traditional FPM, the Gauss antenna radiation pattern is introduced to modify the FPM model. The hybrid method PO + MEC is applied to calculate the scattering from facets and edges of target. The characteristics of coupling field from super-low altitude aircraft target above different terrestrial environments are analyzed by modified FPM. The influences of height of target, RMS and incident angle on coupling field characteristics are discussed. Four important conclusions are obtained: firstly, if the seeker detects target at the Brewster angle, the multipath interference will be weakened in azimuth distribution; secondly, with the increase of RMS, the coupling field of almost angles decreases obviously; thirdly, with the increase of target height, the coupling field of almost angles decreases gradually; fourthly, with the increase of target height, the corresponding angle of minimum value for coupling field decreases. The simulation results can be used for reference in detection for super-low altitude target and optimization for radar system.

\section{Conflicts of Interest}

The authors declare no conflicts of interest regarding the publication of this paper.

\section{References}

[1] Bao, R., Wang, A.Q. and Huang Z.X. (2017) Wide-Band EM Scattering from Multiple Dielectric Targets above a Dielectric Rough Sea Surface Based on Maehly Approximation. 7 th IEEE International Symposium on Microwave, Antenna, Propagation, and EMC Technologies, October 2017, 103-105.

[2] Behdani, M., Dehkhoda, P., Tavakoli, A., et al. (2017) Electromagnetic Scattering from a pec Target over a Random Rough Sea Surface Using Hybrid KA-PO-PTD Method. IEEE International Symposium on Antennas and Propagation \& USNC/URSI Na- 
tional Radio Science Meeting, July 2017, 2045-2046. https://doi.org/10.1109/APUSNCURSINRSM.2017.8073065

[3] Zou, G.X., Tong, C.M., Sun H.L., Song, T., et al. (2017) Electromagnetic Scattering from Simple Target above Composite Rough Surface of Ground and Near Sea in Adjacent Region. International Applied Computational Electromagnetics Society Symposium, August 2017, 1-2.

[4] Behdani, M., Tavakoli, A., Dehkhoda, P., et al. (2016) Electromagnetic Scattering from a PEC Target Buried beneath a Rough Surface Using KA-PO Method. 8th International Symposium on Telecommunications, September 2016, 424-427. https://doi.org/10.1109/ISTEL.2016.7881855

[5] Peng, P., Guo, L.X. and Tong, C.M. (2016) A Study of Multipath Scattering from Low-Altitude Target above Complex Ocean Surface. 11 th International Symposium on Antennas, Propagation and EM Theory, October 2016, 337-339.

https://doi.org/10.1109/ISAPE.2016.7833994

[6] Jonson, J.T. (2001) A Study of the "Four-Path" Mode for Scattering from an Object above a Half-Space. Microwave Optical Technology Letters, 2001, 30, 130-134. https://doi.org/10.1002/mop.1242

[7] Jonson, J.T. (2002) A Numerical Study of Scattering from an Object above a Rough Surface. IEEE Trans. Antennas and Propagation, 50, 1361-1367.

https://doi.org/10.1109/TAP.2002.802152

[8] Zou, G.X., Tong, C.M., et al. (2016) Research on Electromagnetic Scattering Characteristics of Strait Composite Random Rough Surface Based on Improved Hybrid SBR/PO Method. IEEE International Symposium on Antennas, Propagation and EM Theory, October 2016, 587-591.

[9] Michaeli, A. (1984) Equivalent Edge Currents for Arbitrary Aspects of Observation. IEEE Transactions on Antennas and Propagation, 32, 252-258. https://doi.org/10.1109/TAP.1984.1143303 